\title{
BMJ Open Impact of fixed implant supported prostheses in edentulous patients: protocol for a systematic review
}

\author{
Carolina S López, ${ }^{1}$ Constanza H Saka, ${ }^{1}$ Gabriel Rada, ${ }^{2,3,4}$ Daniela D Valenzuela ${ }^{5}$
}

To cite: López CS, Saka CH, Rada G, et al. Impact of fixed implant supported prostheses in edentulous patients: protocol for a systematic review. BMJ Open 2016;6: e009288. doi:10.1136/ bmjopen-2015-009288

- Prepublication history for this paper is available online To view these files please visit the journal online (http://dx.doi.org/10.1136/ bmjopen-2015-009288)

Received 1 July 2015 Revised 26 October 2015 Accepted 24 November 2015

\section{CrossMark}

${ }^{1}$ Faculty of Medicine, Pontificia Universidad Católica de Chile ${ }^{2}$ Department of Internal Medicine, Faculty of Medicine, Pontificia Universidad Católica de Chile ${ }^{3}$ Evidence-based Health Care Program, Faculty of Medicine, Pontificia Universidad Católica de Chile ${ }^{4}$ Epistemonikos foundation, Santiago, Chile

${ }^{5}$ Centro de rehabilitación oral avanzada e implantología (CRAI), Universidad de Concepción, Santiago, Chile

Correspondence to Dr G Rada; radagabriel@epistemonikos. org

\section{ABSTRACT}

Introduction: Edentulism is a debilitating and irreversible condition described as the 'final marker of disease burden for oral health'. Therapy with dental implants is being used on a large scale to replace missing teeth and to rehabilitate edentulous patients with overdentures and implant supported fixed dentures as a method of solving the problem of instability and lack of retention associated with conventional removable prostheses. Fixed implant supported prostheses are an alternative for implant rehabilitation treatment that allow patients to have new fixed teeth. They can be indicated in partial or total edentulous patients, and they can replace single teeth, or teeth and supporting tissues (hybrid prosthesis). They overcome the limitations of conventional dentures, increasing stability and retention, providing functional and psychological advantages for the patients.

Methods and analysis: We will electronically search for randomised controlled trials evaluating the effects of fixed implant supported prostheses in edentulous patients in the following databases: Pubmed/MEDLINE, EMBASE and the Cochrane Central Register of Controlled Trials. We will also try to obtain literature screening references of included studies, searching for trial protocols in the WHO International Clinical Trials Registry Platform, reviewing International Team for Implantology conference proceedings and searching for non-published studies through Open Gray. Two researchers will independently undertake selection of studies, data extraction and assessment of the quality of the included studies. Data synthesis and subgroup analyses will be performed using special Review Manager software. Data will be combined in a metaanalysis using a random effects model.

Results: The results will be presented as risk ratios for dichotomous data, and as mean difference or standardised mean difference for continuous data.

Ethics and dissemination: No ethics approval is considered necessary. The results of this study will be disseminated via peer reviewed publications and social networks.

Trial registration number: CRD42015022086.

\section{INTRODUCTION}

\section{Description of the condition}

Edentulism is a debilitating and irreversible condition described as the "final marker of

\section{Strengths and limitations of this study}

- This will be the first systematic review to assess the impact of implant supported prostheses in edentulous patients on their oral health, quality of life and satisfaction.

- This review aims to provide a high quality synthesis of current evidence for patients and clinicians about this question.

- The main limitation is the heterogeneity of studies available on the subject, and the subsequent possibility to group them to analyse the evidence on each outcome of interest that we have defined.

disease burden for oral health'. Although the prevalence of complete tooth loss has declined over the past decade, it remains a major disease worldwide, especially among older adults. ${ }^{1}$ Many factors influence the prevalence of edentulism between different countries and regions, such as education, economic status, access and opportunity to oral healthcare, lifestyle and attitudes to dental care. Edentulism can lead directly to impairment, functional limitation, and physical, psychological and social disability, ${ }^{2}$ affecting the general and oral health of individuals and, therefore, their quality of life.

Complete denture therapy has become the conventional and most prescribed treatment option for edentulous patients, providing improved aesthetics and function. ${ }^{3}$ However, bone resorption of the alveolar ridge, as a consequence of edentulism, can cause a lack of denture stability and retention, especially for the lower prosthesis, reducing patient comfort, chewing, phonetics and aesthetics. Patients wearing conventional removable prostheses often complain about the instability of their dentures. This leads to a feeling of insecurity, inefficient mastication and overall dissatisfaction with their prosthesis. ${ }^{4}$

Therapy with dental implants is being used on a large scale to replace missing teeth and 
to rehabilitate edentulous patients with overdentures and implant supported fixed dentures. ${ }^{3}$ Placement of endosseous dental implants to retain and support overdentures or fixed complete dentures has been developed as a method for solving the problem of instability and lack of retention associated with conventional removable prostheses. ${ }^{4}$ Improvement in retention and stability of the prosthetic rehabilitation through implant supported prostheses leads to enhanced speaking, swallowing and mastication ability and, therefore, improves patient comfort and satisfaction.

\section{Description of the intervention}

Dental implants represent a proven treatment modality for restoration of edentulous jaws. ${ }^{5}$ Several factors play a role in the decision between fixed and removable implant dentures, such as inter-foraminal space, inter-jaw relationship, oral hygiene, costs and patient preferences. ${ }^{6}$

Fixed implant supported prostheses are an alternative to implant rehabilitation treatment that allow patients to have new fixed teeth. They can be indicated in partial or total edentulous patients and they can replace single teeth, or teeth and supporting tissues (hybrid prosthesis). Selection of which type depends on the patient bone volume and soft tissue conditions ${ }^{7}$ in addition to economic factors and treatment cost. Fixed full arch implant supported prostheses may be either screw or cement retained and are indicated in the presence of enough bone and inter-arch space. ${ }^{6}$ Clinical factors that may prohibit a fixed restoration are mostly related to bone or soft tissues deficiencies. In this instance, rehabilitation can be done by hybrid prostheses.

Hybrid prostheses are fixed rehabilitation prostheses composed of a metal based framework covered with complete denture components (resin and denture teeth), which is screwed onto the implants or the abutments. This treatment modality allows patients to have a completely fixed prosthesis, which can only be removed by a dental professional. ${ }^{89}$

\section{How the intervention might work}

Fixed implant restorations are totally implant supported, with no transference of load to denture bearing areas, thus avoiding the possibility of further resorption, associated with tissue borne prostheses. ${ }^{7}$ They also overcome the limitations of conventional dentures, increasing stability and retention of the prosthetic rehabilitation and thus improving speaking, swallowing and chewing efficiency. This brings functional and psychological advantages for the patients, enhancing their comfort and self-confidence.

\section{Why it is important to do this review}

Previous systematic reviews have addressed the impact of fixed prostheses in partial or total edentulous patients in terms of survival and success. ${ }^{5}{ }^{10-14}$ However, none of these reviews has assessed the subjective effects of fixed implant supported prostheses in edentulous patients. Therefore, we not only aim to update existing reviews, but also to supplement existing evidence by incorporating other impact indicators, such as satisfaction and quality of life.

\section{Objectives}

Our main objective is to evaluate the effects of fixed implant supported prostheses in edentulous patients and to assess their impact on satisfaction and quality of life compared with conventional prostheses or overdentures.

\section{METHODS}

Criteria for considering studies for this review

Types of studies

We will include randomised controlled trials.

\section{Types of participants}

We will include studies examining healthy edentulous patients or with stabilised conditions.

\section{Type of interventions}

The interventions of interest are full arched fixed prosthesis and hybrid prosthesis. We will not restrict by type and number of implants or attachment system.

Comparisons of interest are conventional prostheses and implant supported or implant retained overdentures.

\section{Types of outcomes}

- Primary outcomes

- Patient satisfaction

- Quality of life

- Secondary outcomes

- Clinician's appraisals for treatment success

- Cost

- Technical complications

\section{Search methods for identification of studies} Electronic searches

We will search MEDLINE, EMBASE and the Cochrane Central Register of Controlled Trials with no language or publication restrictions.

The following strategy will be used to search MEDLINE (PubMed):

\#1 "Dental Prosthesis, Implant-Supported" [Mesh]

\#2 (Dental OR denture*) AND Prosthes* AND ((Implant NEAR Supported) OR Implant-Supported) \#3 (Dental OR denture*) AND Prosthes* AND ((Implant NEAR fixed) OR Implant-fixed)

\#4 (Dental OR denture*) AND Prosthes* AND ((fullarch NEAR fixed) OR full-arch)

\#5 (Dental OR denture*) AND Prosthes* AND (hybrid)

\#6 \#1 OR \#2 OR \#3 OR \#4 OR \#5

\#7 "Mouth, Edentulous" [Mesh]

\#8 "Jaw, Edentulous"[Mesh]

\#9 toothless [tiab]

\#10 edentul*[tiab] 
\#11 \#7 OR \#8 OR \#9 \#10

\#12 randomized controlled trial [pt]

\#13 controlled clinical trial [pt]

\#14 randomized [tiab]

\#15 placebo [tiab]

\#16 randomly [tiab]

\#17 trial [tiab]

\#18 groups [tiab]

\#19 \#12 OR \#13 OR \#14 OR \#15 OR \#16 OR \#17 OR \#18

\#20 animals [mh] NOT humans [mh]

\#21 \#19 NOT \#20

\#22 \#6 AND \#11 AND \#21

The MEDLINE strategy will be adapted to the syntax and subject headings of the other databases.

The electronic search will be supplemented by:

- Reviewing for additional relevant studies in the references of the included studies.

- Searching for trial protocols through the WHO International Clinical Trials Registry Platform.

- Searching in International Team for Implantology conference proceedings.

- Searching for unpublished studies through Open Grey.

\section{Data collection and analyses}

De-duplicated results from the literature will be uploaded to Covidence software (http://www.covidence. org).

\section{Selection of studies}

Two authors will independently screen the titles and abstracts yielded by the search against the inclusion criteria. We will obtain full reports for all titles that appear to meet the inclusion criteria or where there is any uncertainty. We will seek additional information from study authors where necessary to resolve questions about eligibility. We will resolve disagreement through discussion. We will record the reasons for excluding trials.

\section{Extraction and management of data}

Using standardised forms, two reviewers will extract data independently from each included study. To ensure consistency, we will conduct calibration exercises before starting the review. Data abstracted will include demographic information, methodology, population and intervention, comparison and outcome details.

We will resolve disagreements by discussion, and one arbitrator will adjudicate unresolved disagreements.

\section{Risk of bias in individual studies}

Two reviewers will independently assess risk of bias using the Cochrane Collaboration tool for assessing risk of bias $^{15}$ which considers random sequence generation, allocation concealment, blinding of participants, personnel and outcomes, incomplete outcome data, selective outcome reporting and other sources of bias. A judgment will be made from the extracted information, rated as 'high risk' or 'low risk'.

If there is insufficient detail reported in the study, we will judge the risk of bias as 'unclear' and the original study investigators will be contacted for more information. Disagreements will be resolved first by discussion and then by consulting a third author for arbitration. We will compute graphic representations of potential bias within and across studies using RevMan 5.1 (Review Manager 5.1).

\section{Measures of treatment effects}

For dichotomous outcomes we will express the estimate of treatment effect of an intervention as risk ratios (RR) (improved patient satisfaction/not) together with $95 \%$ CIs. For continuous outcomes we will use mean difference and SD to summarise the data and 95\% CIs. Where continuous outcomes are measured using different scales, the treatment effect will be expressed as a standardised mean difference (SMD) with 95\% CI.

\section{Dealing with missing data}

Where possible, we will attempt to contact the original authors of the study to obtain any missing data. If important numerical missing data cannot be obtained, an imputation method will be used.

\section{Assessment of heterogeneity}

We will assess the variations in treatment effect from the different trials by means of a formal statistical test (Q statistic) and the $\mathrm{I}^{2}$ statistic. We will consider heterogeneity statistically significant if the $\mathrm{p}$ value is $<0.1$. A rough guide to the interpretation of the $\mathrm{I}^{2}$ statistic given in the Cochrane Handbook is: $0-40 \%$ might not be important, 30-60\% may represent moderate heterogeneity, 50-90\% may represent substantial heterogeneity and $75-100 \%$ considerable heterogeneity.

\section{Data synthesis}

We will only conduct a meta-analysis if there are studies sufficiently homogeneous in terms of design, population, interventions and comparators reporting the same outcome measures.

The results for clinically homogeneous studies will be meta-analysed using the Review Manager Software (RevMan 2011). Meta-analysis will be conducted using the inverse variance method. A random effect model will be used. Separate meta-analyses will be presented for specific populations or interventions if statistically significant heterogeneity is explained by some of these, or if a convincing subgroup effect is found.

For any outcomes where insufficient data are found for a meta-analysis, a narrative synthesis will be presented.

Subgroup analysis and investigation of heterogeneity

The following subgroups will be investigated, if possible: 
- Age $(<60$ vs $>60$ years $)$

- Gender

- Socioeconomic status

\section{Assessment of reporting biases}

We will investigate the presence of publication bias visually with the use of funnel plots. We will base evidence of asymmetry on $\mathrm{p}<0.10$, and present intercepts with $90 \%$ CIs. Other reporting biases, including outcome reporting bias, will be evaluated through discrepancies between the registered protocol and the final publication. If we cannot find the record of a study in the WHO International Clinical Trials Registry Platform, we will contact the authors for more information.

\section{Sensitivity analysis}

We will use sensitivity analysis to assess the impact on the overall treatment effects of inclusion of trials which do not report an intention to treat analysis, have high rates of participant attrition or with other missing data.

\section{Confidence in cumulative evidence}

The quality of evidence for all outcomes will be judged using the Grading of Recommendations Assessment, Development and Evaluation working group methodology (GRADE Working Group). ${ }^{16}$ The certainty of evidence will be assessed across the domains of risk of bias, consistency, directness, precision and publication bias. Certainty will be adjudicated as high, moderate, low or very low.

Acknowledgements Maria Teresa Olivares contributed to devising the search strategy for MEDLINE.

Contributors GR is the guarantor. CHS and CSL drafted the manuscript. DDV is the technical advisor on implant supported prostheses. All authors contributed to the development of the manuscript. All authors devised the MEDLINE search strategy. All authors read, provided feedback and approved the final manuscript.

Competing interests None declared.

Provenance and peer review Not commissioned; externally peer reviewed.

Open Access This is an Open Access article distributed in accordance with the Creative Commons Attribution Non Commercial (CC BY-NC 4.0) license, which permits others to distribute, remix, adapt, build upon this work noncommercially, and license their derivative works on different terms, provided the original work is properly cited and the use is non-commercial. See: http:// creativecommons.org/licenses/by-nc/4.0/

\section{REFERENCES}

1. Emami E, de Souza RF, Kabawat M, et al. The impact of edentulism on oral and general health. Int J Dent 2013;2013:498305.

2. Locker D. Measuring oral health: a conceptual framework Community Dent Health 1988;5:3-18.

3. Caetano CR, Consani RLX, Bacchi A, et al. Influence of different flask systems on tooth displacement and framework misfit in mandibular fixed implant-supported complete dentures. J Prosthodont Res 2013;57:213-18.

4. Prithviraj D, Madan V, Harshamayi P, et al. A comparison of masticatory efficiency in conventional dentures, implant retained or supported overdentures and implant supported fixed prostheses: a literature review. J Dent Implant 2014;4:153-7.

5. Papaspyridakos $P$, Mokti M, Chen CJ, et al. Implant and prosthodontic survival rates with implant fixed complete dental prostheses in the edentulous mandible after at least 5 years: a systematic review. Clin Implant Dent Relat Res 2014;16: 705-17.

6. Barão VAR, Delben JA, Lima J, et al. Comparison of different designs of implant-retained overdentures and fixed full-arch implant-supported prosthesis on stress distribution in edentulous mandible-a computed tomography-based three-dimensional finite element analysis. J Biomech 2013;46:1312-20.

7. Bedrossian E, Sullivan RM, Fortin Y, et al. Fixed-prosthetic implant restoration of the edentulous maxilla: a systematic pretreatment evaluation method. J Oral Maxillofac Surg 2008;66:112-22.

8. Preciado A, Del Río J, Lynch CD, et al. A new, short, specific questionnaire (QoLIP-10) for evaluating the oral health-related quality of life of implant-retained overdenture and hybrid prosthesis wearers. J Dent 2013;41:753-63.

9. Kwon T, Bain PA, Levin L. Systematic review of short- (5-10 years) and long-term (10 years or more) survival and success of full-arch fixed dental hybrid prostheses and supporting implants. J Dent 2014;42:1228-41.

10. Kwon T, Bain P, Levin L. Systematic review of short- (5-10 years) and long-term (10 years or more) survival and success of full-arch fixed dental hybrid prostheses and supporting implants. J Dent 2014;42:1228-41.

11. Pjetursson BE, Thoma $D$, Jung $R$, et al. A systematic review of the survival and complication rates of implant-supported fixed dental prostheses (FDPs) after a mean observation period of at least 5 years. Clin Oral Implants Res 2012:22-38.

12. Lambert FE, Weber H-P, Susarla SM, et al. Descriptive analysis of implant and prosthodontic survival rates with fixed implant-supported rehabilitations in the edentulous maxilla. J Periodontol 2009;80:1220-30.

13. Bozini T, Petridis $\mathrm{H}$, Garefis $\mathrm{K}$, et al. A meta-analysis of prosthodontic complication rates of implant-supported fixed dental prostheses in edentulous patients after an observation period of at least 5 years. Int J Oral Maxillofac Implants 2011;26:304-18.

14. Papaspyridakos $P$, Chen C-J, Chuang S-K, et al. A systematic review of biologic and technical complications with fixed implant rehabilitations for edentulous patients. Int J Oral Maxillofac Implants 2012;27:102-10.

15. Higgins JPT, Altman DG, Sterne JAC. Chapter 8: Assessing risk of bias in included studies. In: Higgins JPT, Green S, eds. Cochrane Handbook for Systematic Reviews of Interventions V.5.1.0. (updated March 2011). The Cochrane Collaboration, 2011. http://www. cochrane-handbook.org

16. The GRADE Working Group. List of GRADE working group publications and grants. http://www.gradeworkinggroup.org/index. htm (accessed 18 Jun 2015) 